\title{
Dynamic FPAA-based Mixed-Signal Processing Circuit for Thin-Film CdTe/Lead-Free Perovskite Photodetectors
}

\author{
Ivailo M. Pandiev ${ }^{1, *}$, Mariya P. Aleksandrova ${ }^{2}$ \\ ${ }^{l}$ Department of Electronic Engineering, Technical University of Sofia, \\ $8 \mathrm{Kl}$. Ohridski Blvd., 1000 Sofia, Bulgaria \\ ${ }^{2}$ Department of Microelectronics, Technical University of Sofia, \\ $8 \mathrm{Kl}$. Ohridski Blvd., 1000 Sofia, Bulgaria \\ ipandiev@tu-sofia.bg
}

\begin{abstract}
New photodetector structure combining thinned CdTe film with lead-free perovskite photoelectric film was produced and investigated. This setting of the CdTe thickness results in photodetector parameters' competitiveness to the state-of-the-art in the field of advanced photoelectric materials. The device shows a promising sensitivity of $\sim 40 \mu \mathrm{A} / \mathrm{W}$, maximum responsivity of $10.6 \mathrm{~mA} / \mathrm{W}$ at $460 \mathrm{~nm}$, equal rise and fall times of $30 \mathrm{~ms}$, and high linearity (maximum linearization error is less than $0.6 \%$ ). However, the optoelectronic performance of CdTe/lead-free perovskite structures integrated with signal processing circuit remains unexplored. For this purpose, Field Programmable Analogue Array (FPAA)-based mixed-signal processing circuit is developed for pulse width modulated electrical signal with duty cycle controlled by the illumination degree of the detecting photoelement. This novel approach guarantees a smooth change of the electrical output at a smooth change of the input illumination between the light and dark switching states and can be practically applied as a precise position detector of moving objects. The paper represents a synergistic connection between microelectronics, electronics, and signal technology.
\end{abstract}

Index Terms-Amplifiers; CdTe; FPAA; Lead-free Perovskites; Photodetectors; Pulse width modulation converters; Thin films.

\section{INTRODUCTION}

In the last few years, an unprecedented growth of interest in organic-inorganic halide perovskite-based solar cells has been observed due to their distinct combination of high efficiency and easy preparation [1]-[4]. However, their stability is still an issue, especially as related to the novel lead-free perovskites, which elemental composition is in line with the requirements for environmentally friendly manufacturing. On the other side, the well-studied CdTebased solar cells exhibit long-term stability of the electrical characteristics due to their photochemical stability. Unfortunately, the films from CdTe usually consist of pinholes, and for this reason, its thickness cannot be decreased to smaller than $4 \mu \mathrm{m}-5 \mu \mathrm{m}$. For the advanced

Manuscript received 16 February, 2021; accepted 30 March, 2021.

This research was supported by the Bulgarian National Science Fund under Grant No. KP06-India-6 under the program for bilateral cooperation Bulgaria-India 2018. thin-film low-cost technology of photoelectric transducers (detectors or solar cells), this is a large thickness and must be decreased, because it limits the cell miniaturization and results in a cost-inefficient device. It also limits the device flexibility (when a flexible substrate is necessary to be used), because of the long-time sputtering process leading to plastic substrate overheating and thermal damage. Therefore, a suitable engineering solution that provides a thin, but pinhole-free absorbing layer together with lower fabrication cost and without compromise to the cell's stability is welcome. Advanced materials engineering recently allowed the synthesis of lead-free perovskite materials with satisfying efficiency compared to the leadhalide ones [5]. They are dispersed in solvent forming solutions with specific viscosity that can be deposited by simple and cheap techniques like spin or spray coating. At the same time, they can have very similar electrophysical properties in terms of energy level alignment and bandgap to the conventional metal telluride and sulfide films, copperindium-selenium (CIS) or copper indium gallium selenide (CIGS) multilayer stacks [6].

In the present paper, a novel design of photoelectric element that implements lead-free perovskite material in combination with thinned CdTe coating with an application as thin-film photodetectors is proposed. In this device, the reduced thickness of the absorbing CdTe can be compensated by an additional film grown from the liquid phase (perovskite), causing densification of the pinholes containing structure of the absorber. The resulting optoelectrical performance is compatible with this one of the pure CdTe thick film photodetector, giving the advantage of the same electrical performance and stability, but achieved with a thinner and lighter structure produced at a lower cost. Although the demonstration of the photodetector's working ability was made for glass-based device, the proposed technology is suitable for flexible substrates as well due to the lower thermal load during the telluride thin film sputtering and the subsequent low-temperature spin coating of the lead-free perovskite film. The resulting photodetector, however, suffers from lower current generated at the same light intensity as compared with the thick non-perovskite 
structures, as well as from lower internal resistance. Then, for the practical applicability of the produced detector, specific measures have to be taken to guarantee the impedance matching between the sensing elements and the sensor signal processing circuit. For full and reliable energy transfer through this interface, it is necessary to design a suitable processing circuit that can work at these specific conditions. Field Programmable Analogue Array (FPAA)based circuit is a suitable solution due to the possibility for dynamic variation of the circuit parameters following the variation of the input signal's amplitude and the level of the input noise voltages and noise currents [7]. Besides, the usage of reconfigurable integrated circuits allows designing a fully integrated device without any external discrete components. This is the reason for the choice of such kind of programmable circuit in the present study to realize a photodetector with an optically controlled variation of a pulsed electrical signal. FPAAs have been widely used in many kinds of microsensors, including MEMS seismic sensor [8], pH monitoring system [9], speech detectors [10], for emulation of photovoltaic I-V curves [11], and as a control circuit for XYZ scanner [12]. However, their application with thin-film photodetectors, in particular such ones with small internal resistance, has not been demonstrated so far.

In this paper, the optoelectric characteristics of the novel photoelectric microelement and the effect of lighting and dimming on the duty cycle of the output pulse electrical signal were showed. In this way, the concept of a new type of photodetector was proven and its practical applicability was demonstrated using an especially realized circuit for electrical signals processing. The data about the device behavior (sensitivity, response time) are of great scientific importance to estimate the stability of the newly synthesized substances incorporated in the photodetector's structure. To the best of our knowledge, this is the first time that an FPAA-based processing circuit integrated with $\mathrm{CdTe} / \mathrm{lead}$ free perovskite photodetector has been investigated.

\section{FABRICATION OF THIN-FILM CD/TE/LEAD-FrEE PEROVSKITE PHOTODETECTORS}

The proposed photodetector structure is shown in Fig. 1(a). Glass substrate was coated with $\mathrm{ZnO}$ doped by gallium (GZO) film, serving as a transparent front electrode (cathode) and heat mirror. The electron transporting layer (ETL) from $\mathrm{TiO}_{2}$ was subsequently grown, followed by CdTe film, methylammonium tin iodide-based lead-free perovskite film (both as absorbers), and CsBr-based quantum dots containing film as a hole transporting layer (HTL). Finally, a gold contact is deposited, providing negligible contact resistance (ohmic contact) at the interface with the HTL and back reflection of the highly energetic photons into the structure for enhanced and full absorption of the light.

Conventional microfabrication technologies were used for the growth of the individual layers. The glass substrates were cleaned in a standard detergent solution, including ammonia, hydrogen peroxide, and distilled water in the ratio $1: 1: 3$. Thin films of GZO were prepared by radiofrequency (RF) sputtering of a 3 inches diameter target on the glass substrates. The sputtering voltage was kept constant and equal to $0.7 \mathrm{kV}$ for the GZO films (plasma power of $112 \mathrm{~W}$ and power density of $23 \mathrm{~W} / \mathrm{inch}^{2}$ ). The total sputtering pressure in the chamber (argon) was $1 \times 10^{-3}$ Torr without additional oxidation. The thickness of the GZO film was $100 \mathrm{~nm}$. $\mathrm{TiO}_{2}$ film was then sputtered at a voltage of $1 \mathrm{kV}$ (plasma power of $160 \mathrm{~W}$ and power density of $33 \mathrm{~W} / \mathrm{inch}^{2}$ ), at a total pressure in the vacuum chamber of $4 \times 10^{-2}$ Torr with additional oxidation of the titanium sputtering target (the oxygen partial pressure was $2 \times 10^{-4}$ Torr), resulting in a $50 \mathrm{~nm}$ thin film as an ETL. CdTe serving as a half of the photoelectric converting layer was RF sputtered on the $\mathrm{TiO}_{2}$ at an argon sputtering pressure of $1 \times 10^{-2}$ Torr and a sputtering voltage of $1 \mathrm{kV}$. The plasma power was $230 \mathrm{~W}$, and the power density was $48 \mathrm{~W} / \mathrm{inch}^{2}$, which resulted in a deposition of layer with a thickness of $2.4 \mu \mathrm{m} . \mathrm{CH}_{3} \mathrm{NH}_{3} \mathrm{SnI}_{3}$ lead-free perovskite ink was spin-coated on the CdTe at $1500 \mathrm{rpm}$ per $30 \mathrm{~s}$ and consequently dried for $10 \mathrm{~min}$ at $130^{\circ} \mathrm{C}$, resulting in a $300 \mathrm{~nm}$ thin film that is the second half of the photoelectric converting layer. CsBr-based quantum dots dispersed in a toluene solution were spincoated at $1000 \mathrm{rpm}$ per $30 \mathrm{~s}$ and consequently dried for $10 \mathrm{~min}$ at $90{ }^{\circ} \mathrm{C}$. Finally, the gold electrodes were deposited by vacuum DC sputtering at a total pressure of $1 \times 10^{-1}$ Torr, plasma current of $30 \mathrm{~mA}$ (plasma power of $30 \mathrm{~W}$ and power density of $14 \mathrm{~W} / \mathrm{inch}^{2}$ ). This final deposition process was conducted through a square shadow mask with a side size of $1.5 \mathrm{~cm}$, defining the active area of the photodetector of $2.25 \mathrm{~cm}^{2}$.

The films' thicknesses were measured by surface scanning Alpha-step Tencor setup. The device testing was conducted with a lab-made solar simulator having a standard light source for photoelectric device testing, consisting of color filters (red, blue, and yellow) for spectral sensitivity determination and a regulating aperture for light intensity control, as shown in Fig. 1(b).

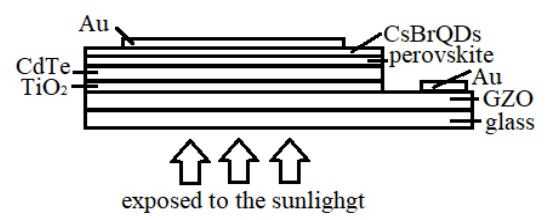

(a)

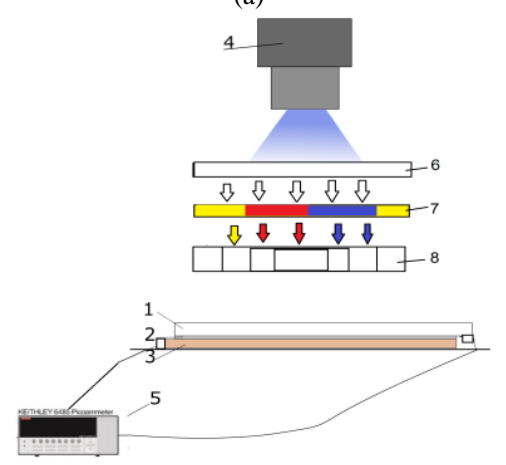

(b)

Fig. 1. Fabrication and testing approach: a) schematic drawing of the photodetector structure; b) experimental setup for measurement of the optoelectric characteristics of the device: 1 - substrate coated with a front electrode, 2 - functional film, 3 - backside electrode, 4 - light source, 5 picoammeter, 6 - switching between lens and diffuser (for directional or scattered light, respectively), 7 - color filter, 8 - light intensity regulating aperture. 
The intensity of the emitted light at each aperture shutter position was determined by a luminance meter Konica Minolta LS110. The DC voltage was recorded by Voltcraft VC 820 voltmeter connected with PC, and the photocurrent was measured by picoammeter Keithley 6485. The shape and the duty cycle of the modulated output signal were observed with a digital oscilloscope Tektronix TDS1002B.

\section{DESIGN OF FPAA-BASED PROCESSING CIRCUIT}

The most widely spread convertors for the photocurrent into voltage are the current-controlled voltage sources (CCVSs) or shunt-feedback transimpedance amplifiers (TIA) with a conventional operational amplifier based on the electronic circuit [13], [14], as was shown in Fig. 2. This electronic circuit is particularly suitable for specific types of thin-film photodetector structures, as it is characterized by a very small input resistance and according to Miller's theorem can be determined as follows

$$
r_{i A} \approx \frac{R_{F}}{1+A_{d 0}} \stackrel{A_{d 0}>>1}{\approx} \frac{R_{F}}{A_{d 0}}<1 \Omega
$$

where $A_{d 0}$ is the open-loop voltage gain of the op amp.

Therefore, the value of the resistance $r_{i A}$ is very small and can be almost always neglected compared to the internal resistance of the input signal source $r_{G}$.

The output resistance of the circuit in Fig. 2 is also very small and can be determined from the formula for a circuit with a parallel voltage negative feedback

$$
r_{O A} \approx \frac{r_{O}}{1+\beta A_{d 0}} \stackrel{\beta A_{d 0}>>1}{\approx} \frac{r_{o}}{\beta A_{d 0}}=\frac{r_{o}}{A_{d 0}}\left(1+\frac{R_{F}}{r_{G}}\right),
$$

where $r_{o}$ is the output resistance of the operational amplifier without feedback, and $\beta=r_{G} /\left(r_{G}+R_{F}\right)$ is the transmission coefficient of the feedback circuit.

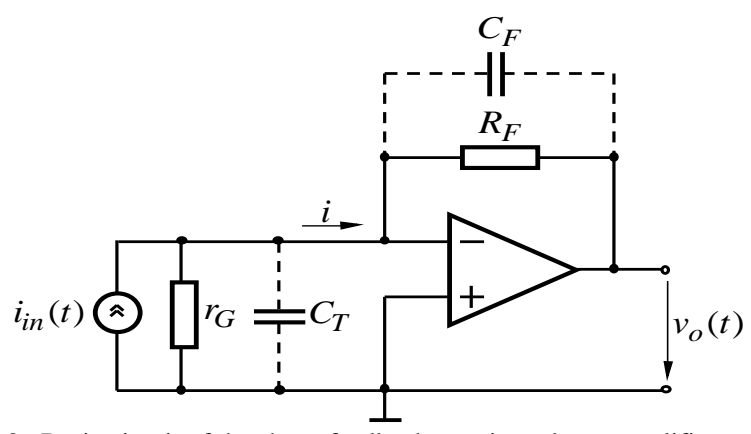

Fig. 2. Basic circuit of the shunt-feedback transimpedance amplifier.

Since the operational amplifiers have a very large input resistance, and accordingly, small input currents, the inverting input is a virtual zero (ground) of the circuit, and the current flowing through the resistor $R_{F}$ is equal to the input current $I$. Then the output voltage is obtained

$$
v_{o}=-R_{F} i
$$

where $i=i_{G}+i_{R_{G}} \approx i_{G}$ for $i_{G}>>i_{R_{G}}$.
When $i_{G}=0$ using a real operational amplifier, the voltage at the output of the circuit from Fig. 2 is not zero. This voltage is formed from the input offset voltage $V_{i o}$ and input bias current $I_{B}^{-}$at the inverting input terminal of the op amp. The value of the output offset voltage $V_{\text {o,err }}$ is obtained from the formula

$$
\begin{gathered}
V_{o, e r r}=V_{o, e r r}\left(U_{i o}\right)+V_{o, e r r}\left(I_{B}^{-}\right)= \\
=\left(1+R_{F} / r_{G}\right) \times\left[V_{i o}+\left(R_{F} \| r_{G}\right) I_{B}^{-}\right],
\end{gathered}
$$

where $V_{o, \operatorname{err}\left(V_{i o}\right)}=\left(1+R_{F} / r_{G}\right) V_{i o}$ and $V_{o, \operatorname{err}\left(I_{B}^{-}\right)}=I_{B}^{-} R_{F}$.

A feedback capacitor $C_{F}$ is connected in parallel to the resistor $R_{F}$ when the circuit works in a wide frequency range mode to limit the high-frequency noise that can occur in the output [15], [16]. If the operational amplifier is considered an ideal active element for the pole frequency and the zero frequency of the reciprocal of the negative feedback coefficient (or Noise Gain - NG coefficient), the following expressions are obtained, respectively:

$$
\begin{gathered}
f_{p}=1 / 2 \pi R_{F} C_{F}, \\
f_{z}=1 / 2 \pi R_{F}\left(C_{F}+C_{T}\right),
\end{gathered}
$$

where $C_{T}=C_{G}+C_{M}+C_{i d}^{-}$is the total input capacitance of the circuit $\left(C_{i d}^{-}=C_{i d}+C_{i C M}^{-}\right.$is the input capacitance at the inverting input of the op amp, $C_{G}$ is the internal capacitance of the photodetector, and $C_{M}$ is the parasitic capacitance with typical values $1 \ldots 3 p F$ ).

Since $f_{z}<f_{p}$ after the zero frequency, the noise gain begins to increase with a rate of $+20 \mathrm{~dB} / \mathrm{dec}$. If the $\mathrm{NG}$ characteristic crosses the amplitude vs. frequency response characteristic of the op amp without feedback $\left|\dot{A}_{d}\right|$ in range with a slope of $-20 \mathrm{~dB} / \mathrm{dec}$, the variation rate of the transmission coefficient is approximately $40 \mathrm{~dB} / \mathrm{dec}$. A total dephasing of $180^{\circ}$ is obtained at the point of intersection of $\left|\dot{A}_{d}\right|$ and $1 /|\dot{\beta}|$, and the circuit becomes unstable because the phase condition for self-excitation is fulfilled. To stabilize the circuit, it is necessary to increase the value of the capacitance $C_{F}$ so that for a frequency equal to $f_{p}$ or less than $f_{p}$ to obtain the intersection between the characteristics of the coefficients $\left|\dot{A}_{d}\right|$ and NG. If $C_{T}>C_{F}$, for the optimal value of the capacitor $C_{F}$, the following dependence is obtained

$$
C_{F, o p t}=\sqrt{\frac{C_{T}}{2 \sqrt{2} \pi R_{F} B_{1}}} \approx \sqrt{\frac{C_{T}}{2 \pi R_{F} B_{1}}} .
$$

Then the high cutoff frequency (or corner frequency) of the circuit at the optimal value of $C_{F}$ can be written as 


$$
f_{h}=f_{p}^{\prime}=\frac{1}{2 \pi R_{F} C_{F, o p t}}=\sqrt{\frac{B_{1}}{2 \pi R_{F} C_{T}}} .
$$

After obtaining a voltage proportional to the photocurrent, the output of the amplifier is often connected to a low-pass filter (LPF) circuit to eliminate the highfrequency interference, and then it is transferred to a signal sampling circuit. The use of an analog-to-digital converter or an analog pulse circuit implemented by a voltage-tofrequency converter improves the noise resistance and increases the conversion accuracy of the photodetector.

Nowadays, the most commonly used analog-to-digital converters (ADCs) are the parallel two-step data converters and successive approximation data converters. For both types of ADC, a digital word is displayed in series with clock synchronization. Additionally, there is a pin for synchronizing the signal, and during its active logical values (zero or one), the data are read by the digital control device, most often a microcontroller. Serial data transmission is preferred for sensors involving a signal processing circuit because a small number of pins are used, and hence a smaller package size is obtained.

Due to the limited physical size of many intelligent sensors, ADCs are often produced with a small resolution, which in some cases affects the accuracy of the conversion. Then, instead of analog-to-digital voltage conversion from the amplifier, voltage-to-frequency conversion is applied. The circuit diagram of a voltage-to-frequency converter is shown in Fig. 3(a), and the sample signals of the input and output waveforms are shown in Fig. 3(b) [17], [18], [19]. The input voltage passes through an anti-aliasing low-pass filter before being applied to the non-inverting input of an analog comparator. Thus, before sampling of the input voltage, the unknown higher-order harmonics that are greater than one-half the sampling frequency can be eliminated.

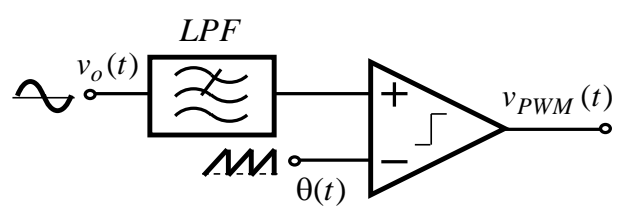

(a)

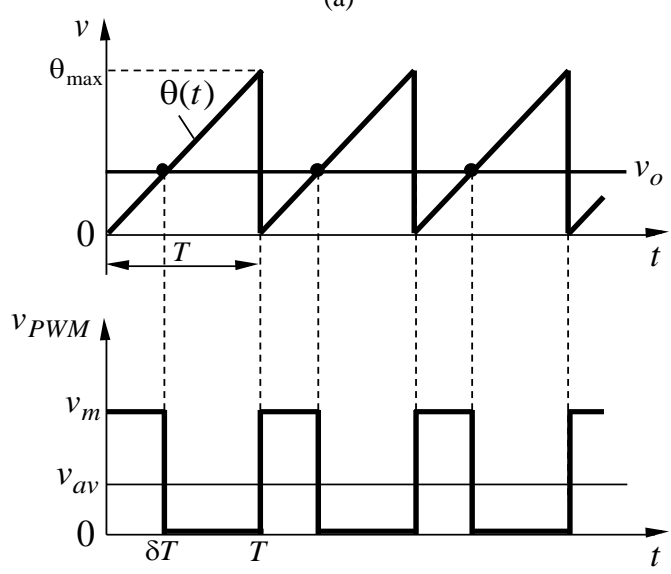

(b)

Fig. 3. Voltage-to-frequency conversion: a) circuit diagram; b) sample signals of the input and output waveforms.

To realize pulse width modulation (PWM), the voltage after the low-pass filter is applied to the non-inverting input of a comparator, and a sawtooth periodic signal is applied to the other input of the comparator. The sawtooth signal can be represented by the following analytical formula

$$
\theta(t)=\frac{\theta_{\max }}{T} t \text { at } 0 \leq t \leq T,
$$

where $T=1 / f$ is the period of a PWM cycle and $\theta_{\max }$ is the amplitude of the sawtooth wave.

In case the signal is obtained from a sawtooth generator with symmetrical or differential output (or with positive and negative components), the analytical formulas are:

$$
\left[\begin{array}{c}
\theta^{+}(\mathrm{t})= \\
=\frac{0.5 \times \theta_{\max }}{\mathrm{T}} \mathrm{t}+\mathrm{V}_{\mathrm{MR}}-\frac{0.5 \times \theta_{\max }}{2} \text {, at } 0 \leq \mathrm{t} \leq \mathrm{T}, \\
\theta^{-}(\mathrm{t})= \\
=-\frac{0.5 \times \theta_{\max }}{\mathrm{T}} \mathrm{t}+\mathrm{V}_{\mathrm{MR}}+\frac{0.5 \times \theta_{\max }}{2} \text {, at } 0 \leq \mathrm{t} \leq \mathrm{T},
\end{array}\right],
$$

where $V_{M R}$ is an offset voltage or signal ground.

As a result, the single-ended signal can be found by

$$
\theta(t)=\theta^{+}(t)-\theta^{-}(t)=\frac{\theta_{\max }}{T} t-0.5 \times \theta_{\max } \text { at } 0 \leq t \leq T \text {. (11) }
$$

According to (11), the single-ended signal will be changed from $-\frac{\theta_{\max }}{2}$ (at $\left.t=0\right)$ to $+\frac{\theta_{\max }}{2} \quad($ at $t=T)$. Therefore, the sawtooth wave is located in the first and fourth quadrants. In case it is necessary to place the signal only in the first quadrant, a constant component with a voltage value equal to $0.5 \times \theta_{\max }$ has to be added (see (9)) . When the voltage $v_{o}$ is greater than $\theta(t)$, a pulse with an amplitude $v_{m}$ is obtained at the output. For $v_{o}<\theta(t)$, the comparator switches and the output voltage becomes zero

$$
v_{P W M}(t)=\frac{1}{T} \int_{0}^{\delta T} v_{m} d t=\delta v_{m},
$$

where $\delta$ is the duty ratio of the PWM cycle.

At a concrete value of the pulse width $t_{p w}$, the average output voltage is equal to $v_{a v}=\delta v_{m}=\left(t_{p w} / T\right) v_{m}$.

For the symmetrical output of the comparator, the positive and negative components are $v_{P W M}^{+}(t)=\frac{\delta v_{m}}{2}+V_{M R}$ and $v_{P W M}^{-}(t)=-\frac{\delta v_{m}}{2}+V_{M R}$, respectively. The resulting single-ended output signal $v_{P W M}(t)=v_{P W M}^{+}(t)-v_{P W M}^{-}(t)$ can be represented according to (12).

Under the design of the mixed-signal processing circuit for voltage to frequency converter, the variations of the parameters of the input signal, such as the amplitude and level of noise voltages or currents, are critical for the stable operation. It means that the implemented circuit has to be 
able to change the transimpedance parameter at variations in the amplitude of the input signal and to provide the ability for adjusting the pole frequency of the LFP at various noise levels. These requirements can be relatively easily achieved by applying the pulse-width modulation technique, using a dynamically programmable FPAA system.

\section{A. FPAA Programming}

The mixed-signal processing circuit, including transimpedance amplifier, 8-bit ADC using the successive approximation register (SAR) circuit and PWM circuit can be implemented by programming in FPAA AN231E04 [20] from ANADIGM. The AN231E04 is characterized by a higher degree of integration, providing the greatest functionality and obtaining the widest bandwidth (up to $4 \mathrm{MHz}$ ) in the range of variation of the input and output signals from $0 \mathrm{~V}$ to $3.3 \mathrm{~V}$ (or $2.8 \mathrm{~V}$ ). Such a device can be programmed by using the ANADIGM DESIGNER 2 software system. A circuit diagram of the FPAA-based mixed-signal processing circuit, including the transimpedance amplifier, is shown in Fig. 4.

As can be seen from Fig. 4, a transimpedance amplifier, low-pass filter, and ADC (or PWM circuit) are required for the implementation of the circuit diagram given in Fig. 2 and Fig. 3(a). Such functional blocks can be relatively easily obtained by using the standard Configurable Analog Blocks (CABs) available in the FPAA AN231E04. Furthermore, the parameters of the selected CABs can be dynamically changed according to the parameters of the input current by using the graphical user interface of the ANADIGM DESIGNER 2 program.

To obtain a voltage-to-frequency converter, first-order low-pass filter modules and an analog comparator were used, comparing the voltage from the amplifier with the voltage from the sawtooth signal generator. The sawtooth signal generator was implemented with the configurable analog module OscillatorSawSqr1. The signal type, frequency, and amplitude had to be set for its work. The generated signal was quartz stabilized, which provided high stability of the produced periodic signal (the relative change of the frequency was within $\pm 10 \mathrm{ppm}$ ).

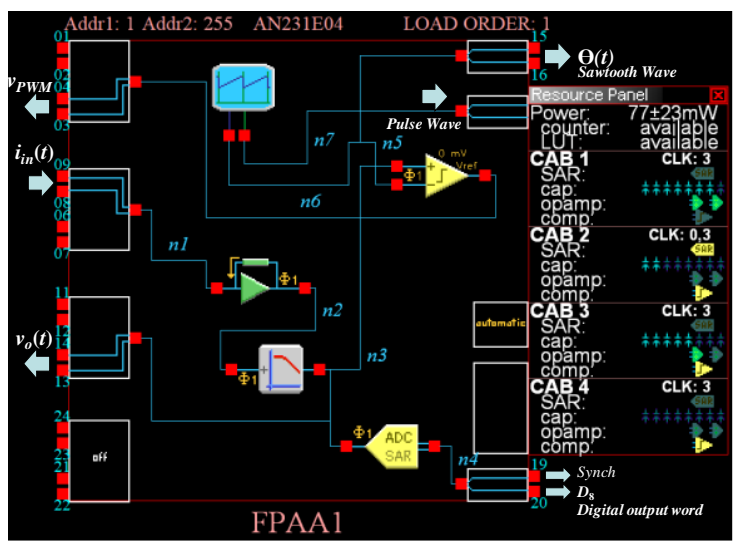

Fig. 4. Mixed-signal processing circuit implemented in FPAA AN231E04. External view of the FPAA configuration in ANADIGM DESIGNER 2 program.

The conversion of the output voltage into a binary number proportional to this voltage is performed by using the configurable analog ADC-SAR, as shown in Table I.
This module produces an 8-bit digital output word Data as a serial sequence synchronized to the CLOCKB clock with a frequency equal to Clock 0 . The Synch bit will switch to logical "1" during the valid data output. When a byte serially outcomes sequentially from the FPAA, the Synch signal is high and each bit of data is accompanied by one CLOCKB clock pulse. Examples of timing diagrams of the Synch, Data, and CLOCKB signals from an analog-todigital converter included in a functional diagram with FPAA are shown in Fig. 5.

The output of this CAM can only be connected to a Type2 IO Cell, and not to the input of any other CAM. The IO Cell has to be configured in Digital Output mode. The Synch and Data signals will be routed to the upper and lower pins of the IO Cell, respectively, as shown in Fig. 4. Each data bit will become valid during the first half of the SAR clock period (CAM CLOCKB) and will be held during the second half of the SAR clock period.

\section{Clock}

(a)

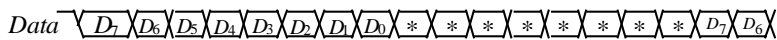

(b)

Synch

(c)

Fig. 5. Example of timing diagrams of: a) Synch; b) Data, c) CLOCKB signals.

Table I summarizes the complete information for the used CABs available in the AN231E04.

TABLE I. CONFIGURABLE ANALOG BLOCKS FOR THE MIXEDSIGNAL PROCESSING CIRCUIT ACCORDING TO THE BLOCK

\begin{tabular}{|c|c|c|c|}
\hline \multicolumn{4}{|c|}{ DIAGRAM IN FIG. 4.} \\
\hline Name & Symbol & Options & Parameters \\
\hline $\begin{array}{c}\text { Transimpedance1 } \\
\text { (Transimpedance } \\
\text { v1.0.3) }\end{array}$ & & $\begin{array}{c}\text { Output: Phase } \\
\text { Phase } 1\end{array}$ & $\begin{array}{c}\text { Transimpe- } \\
\text { dance }[\mathrm{V} / \mu \mathrm{A}] \text { : } \\
0.200\end{array}$ \\
\hline $\begin{array}{c}\text { FilterBilinear1 } \\
\text { (FilterBilinear } \\
\text { v1.0.2) }\end{array}$ & & $\begin{array}{l}\text { Filter Type: } \\
\text { Low-Pass } \\
\text { Input } \\
\text { Sampling: } \\
\text { Phase Phase 1 } \\
\text { Polarity: Non- } \\
\text { inverting }\end{array}$ & $\begin{array}{l}\text { Corner } \\
\text { Frequency: } \\
\text { [kHz] } 1.00 \\
\text { Gain: } 1.00\end{array}$ \\
\hline $\begin{array}{c}\text { OscillatorSawSqr1 } \\
\text { (OscillatorSawSqr } \\
\text { v0.1.1) }\end{array}$ & 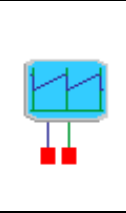 & $\begin{array}{c}\text { Sawtooth } \\
\text { Wave: Rising }\end{array}$ & $\begin{array}{c}\text { Osc. } \\
\text { Frequency } \\
\text { [kHz]: } \quad 10.0 \\
\text { Sawtooth } \\
\text { Peak [V]: } \\
1.00\end{array}$ \\
\hline $\begin{array}{c}\text { Comparator1 } \\
\text { (Comparator } \\
\text { v1.1.1) }\end{array}$ & $=$ & $\begin{array}{c}\text { Output } \\
\text { Polarity: Non- } \\
\text { inverted } \\
\text { Hysteresis: } \\
0 \mathrm{mV} \\
\text { Output Synch: } \\
\text { None }\end{array}$ & none \\
\hline $\begin{array}{c}\text { ADC-SAR1 } \\
(\text { ADC-SAR v1.0.1) }\end{array}$ & $=\underbrace{A D C}_{S A R}=$ & $\begin{array}{c}\text { Input Phase: } \\
\text { Phase } 1\end{array}$ & none \\
\hline
\end{tabular}


The input amplifier Transimpedance1 was used to convert the input current with a transimpedance $z$ equal to $0.2 \mathrm{~V} / \mu \mathrm{A}$. For the oscillator with sawtooth and pulse waves, the oscillation frequency was chosen with an initial value equal to $10 \mathrm{kHz}$ and the sawtooth peak was equal to $1 \mathrm{~V}$. According to the level of voltage that is produced from the transimpedance amplifier, the maximum value can come up to $3 \mathrm{~V}$. Since the current generated by the photodetector is of the order of several microamperes, these parameter values proved to be completely acceptable, being within the linear operating range of the used CAMs.

By routing the electrical connections between the configurable modules and the input/output ports within ANADIGM DESIGNER 2 program, the complete electronic circuit was obtained.

\section{B. Input and Output Stages of the Processing Circuit}

To convert the input current produced by the photodetector, a CAM that creates a transimpedance amplifier (Fig. 6) targeted at photodiode interfacing was used. A transimpedance amplifier CAM [20] converts an input current into an output voltage and the programmable transimpedance parameter sets the ratio of the current to voltage conversion.

The transfer function for this circuit is

$$
v_{o}=-z \times i_{\text {in }},
$$

where $z$ is the transimpedance and $i_{i n}$ is the average input current during the selected output phase. The $i_{\text {in }}$ is half the differential input current because this CAM is designed to interface with a zero-bias photodetector that is conducting $i_{\text {in }}$ both into and out of this CAM. The value of the transimpedance $z$ can be found by the master clock frequency $f_{S}$ and the absolute values of the switched capacitances.

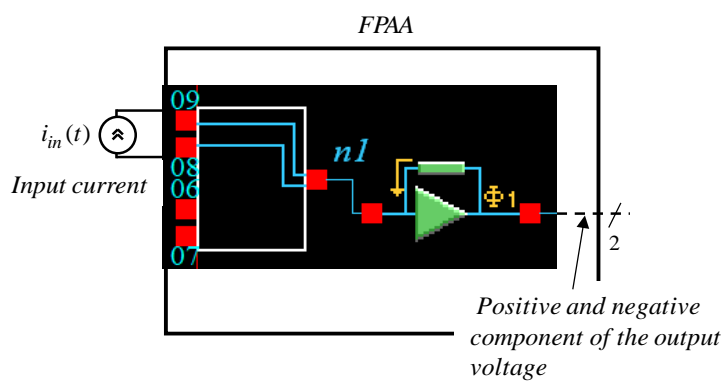

Fig. 6. Circuit of the input transimpedance amplifier for photodetector interfacing.

The output signal of the FPAA was transferred to an integrating differential amplifier (see Fig. 7) implemented with a rail-to-rail input/output op amp MCP6024 (from Microchip Tech.), for which the output voltage in the lowfrequency range was obtained as follows

$$
\begin{gathered}
v_{P W M}=\left.v_{\text {OUT }}^{-}\right|_{v_{\text {OUT }}^{+}=0}+\left.v_{\text {OUT }}^{+}\right|_{v_{\text {OUT }}^{-}=0} \approx \\
\approx-\frac{R_{F}}{R_{N}} v_{\text {OUT }}^{-}+\left(1+\frac{R_{F}}{R_{P}}\right) \times \frac{R_{F}}{R_{F}+R_{P}} v_{O U T}^{+} .
\end{gathered}
$$

Since the condition for resistance balance of the resistors is

$$
\frac{R_{F}}{R_{N}}=\frac{R_{F}}{R_{P}},
$$

(15) is simplified by finding the gain coefficient

$$
A_{V}=\frac{v_{O U T}}{v_{O U T}^{+}-v_{O U T}^{-}} \approx \frac{R_{F}}{R_{N}} .
$$

Since $R_{F}=R_{N}=10 \mathrm{k} \Omega$, the output voltage is equal to the difference of the input voltages or $v_{P W M}=v_{O U T}^{+}-v_{O U T}^{-}$.

In the high-frequency range, the resistance of the capacitors decreases, resulting in a voltage gain limiting. In this case, the high cutoff frequency of the circuit is as follows

$$
f_{h} \approx 1 /\left(2 \pi R_{F} C_{F}\right) \approx 1.6 M H z,
$$

where $C_{F}=5 p F$ is the feedback capacitance of the amplifier.

Then the rise time $t_{r}$ of the output pulse can be found by the following formula

$$
t_{r} \approx 0,35 / f_{h} \approx 200 n s,
$$

which is acceptable according to the parameters of the positive and negative pulse signals and the unity-gain bandwidth of the chosen op amp equal to $10 \mathrm{MHz}$.

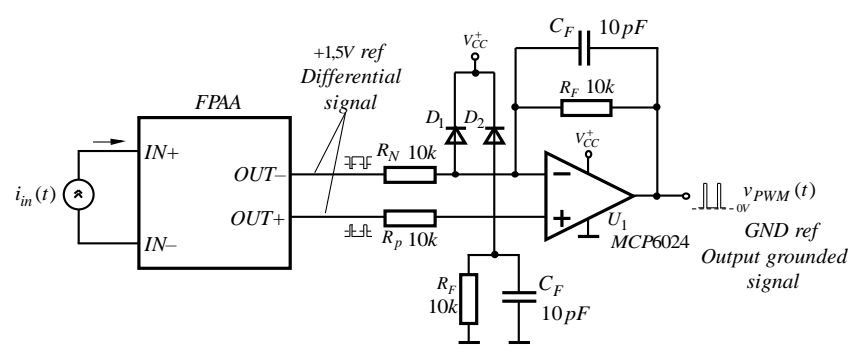

Fig. 7. An electrical circuit of an output integrating differential amplifier.

As a result, the rising edge (or positive edge) of the output pulses for the modulated signal will be obtained without overshoots (or spikes).

\section{RESUlTS AND DisCUSSION}

The response of the photodetector to various light intensities is an important evaluation of the device performance, so the photocurrent and voltage against time were measured for different light intensities ranging from dark to $500 \mathrm{~cd} / \mathrm{m}^{2}\left(73 \mu \mathrm{W} / \mathrm{cm}^{2}\right)$. To demonstrate the workability of the photoelectric transducing structure from Fig. 1(a), the dependences of the produced voltage and current on the light intensity for white light and light colors at a constant intensity of $400 \mathrm{~cd} / \mathrm{m}^{2}$ were shown in Fig. 8(a) and Fig. 8(b), respectively. The maximum intensity chosen for the testing is equivalent to a natural indoor environment with light coming from the outdoor illumination. Therefore, 
an extensive analysis of these relations is not provided and the focus was put on the basic response characteristics of the photodetector to different light stimuli measured according to the setup shown in Fig. 1(b), which are important for the correct work of the FPAA circuit.

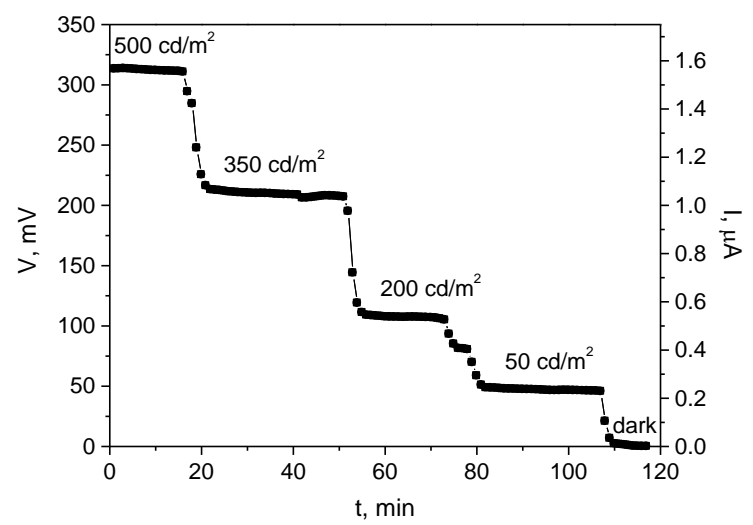

(a)

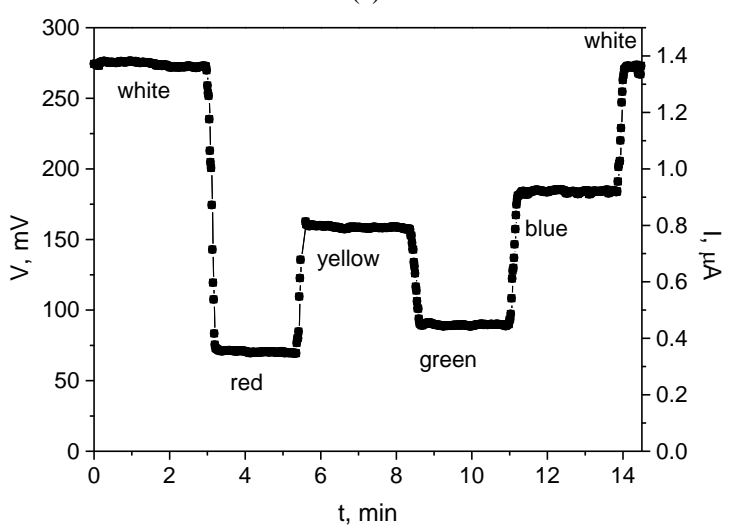

(b)

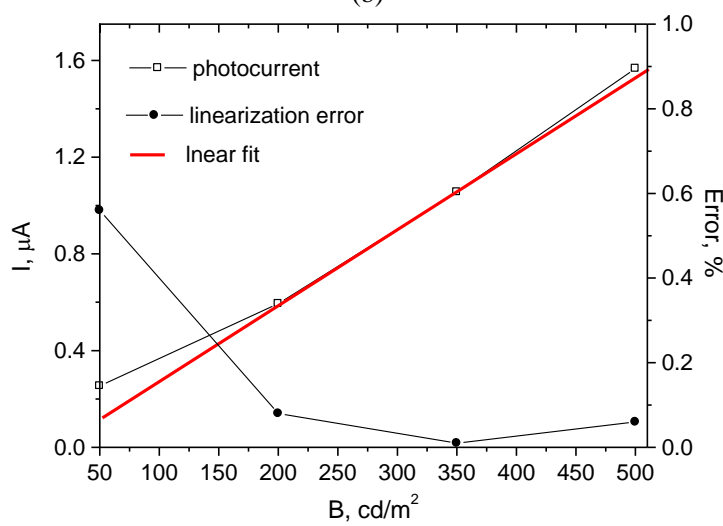

(c)

Fig. 8. Electric response of the produced photodetector at: a) different light intensities of white light; b) different light colors at a constant light intensity of $400 \mathrm{~cd} / \mathrm{m}^{2}$ (spectral response); c) linearity of the photodetector.

From the measured characteristics, it was found that the dark current through the structure was $\sim 15 \mathrm{nA}$. The device exhibited an excellent photoelectric sensitivity to illumination levels near the natural indoor light level, existing without additional illumination from lamp sources, which is an advantage for the future practical applicability of the detector. At white light with an intensity of $500 \mathrm{~cd} / \mathrm{m}^{2}$, a single cell produced a voltage equal to approximately $313 \mathrm{mV}$ and a current of $\sim 1.6 \mu \mathrm{A}$ measured from an area of $2.25 \mathrm{~cm}^{2}$. The transition from photometric to radiometric units showed that the sensitivity was $\sim 40 \mu \mathrm{A} / \mathrm{W}$ and the maximum responsivity was $10.6 \mathrm{~mA} / \mathrm{W}$ at $460 \mathrm{~nm}$, which is quite acceptable value, considering the involved novel lead-free material and the smaller thickness of the CdTe film. For comparison, this value slightly exceeds the recently reported $10 \mathrm{~mA} / \mathrm{W}$ for an advanced graphene-based photodetector [21]. Regarding the spectral sensitivity, favourable behavior was observed at blue color illumination, because the photocurrent drop was the smallest ( $\Delta I=0.45 \mu A$ vs. $\Delta I=1 \mu A$ at red color). It means that even continuously exposed to direct sunlight, the microstructure does not absorb the heating component of the sun, which is emitted in the near-infrared range.

The device non-linearity after a linear fit of the photocurrent was found to be less than $1 \%$, as is shown in Fig. 8(c). The maximum value of the error from the nonlinearity reached $0.56 \%$ at low photocurrents (less than $400 \mathrm{nA}$ ) achieved at low brightness of less than $100 \mathrm{~cd} / \mathrm{m}^{2}$. This value can be ascribed to the noise currents. For light intensity in the range of $200 \mathrm{~cd} / \mathrm{m}^{2}-500 \mathrm{~cd} / \mathrm{m}^{2}$, the error dropped to values smaller than $0.1 \%$, which guarantees a sufficient accuracy, considering the manufacturing tolerances of the parameters of the produced photodetectors.

The rising and falling edges of the photocurrent pulses obtained from the light-to-dark transition (Fig. 9(a)) and vice versa were investigated for estimating the rise time $t_{\text {rise }}$ (Fig. 9(b)) and the fall time $t_{\text {fall }}$ (Fig. 9(c)) of the photodetector. The device exhibited symmetry in its timedependent parameters with an equal rise and fall time of $30 \mathrm{~ms}$. The value is compatible with the $\mathrm{MoS}_{2} /$ Graphene heterojunction-based photodetectors, showing $20 \mathrm{~ms}$ and $3 \mathrm{~ms}$ for the rise and fall time, respectively [22], and operating in a similar spectral range.

To demonstrate the successful integration of the photodetector structure with the proposed FPAA circuit with additional input amplifier and external output stage, as shown in Fig. 6 and Fig. 7, a prototype using the AN231K04-DVLP3 development system built around the FPAA AN231E04 was implemented. For this purpose, two photoelectric cells were connected in parallel. The IC AN231E04 was biased with a single power supply voltage equal to $+3.3 \mathrm{~V}$, and as a result, the produced signal ground voltage was equal to $1.5 \mathrm{~V}$. The experimental setup of the prototype, serving as a proof of concept, is shown in Fig. 10 .

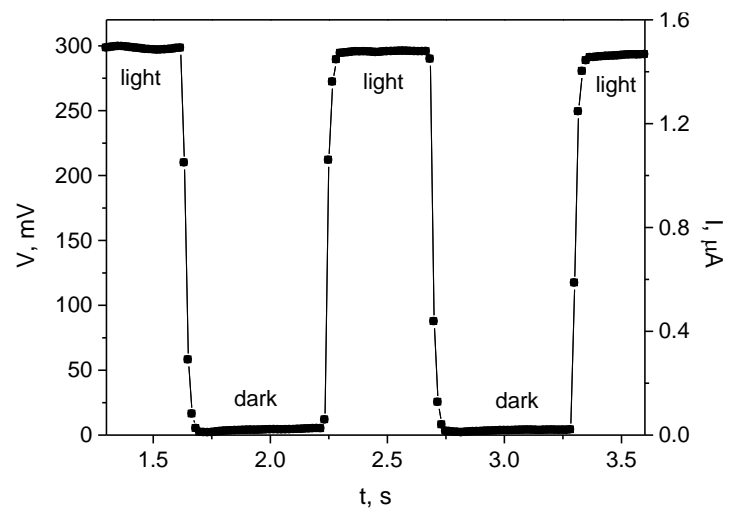

(a) 


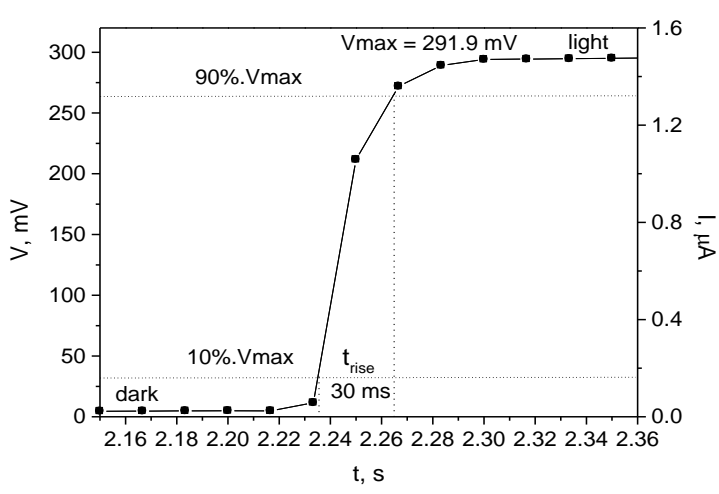

(b)

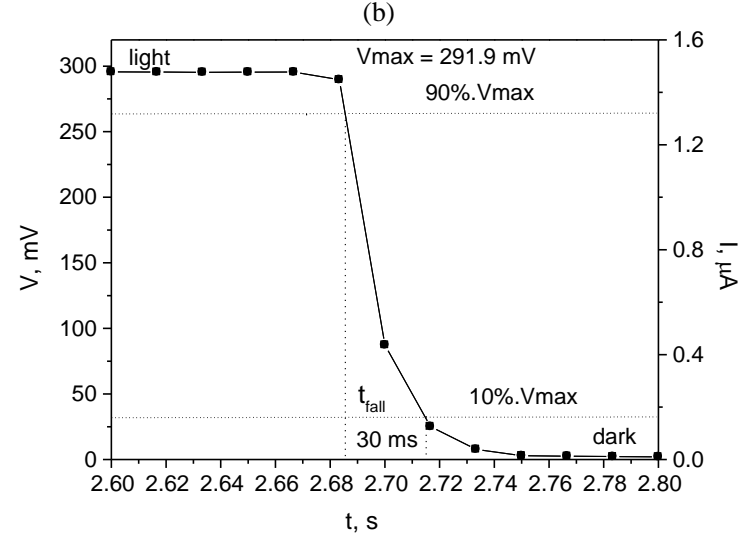

(c)

Fig. 9. Response speed of the produced photodetector to white light with a maximal intensity of $400 \mathrm{~cd} / \mathrm{m}^{2}$ : a) general view of the pulses at light-todark transition and vice versa; b) leading edge of the pulse; $c$ ) trailing edge of the pulse.

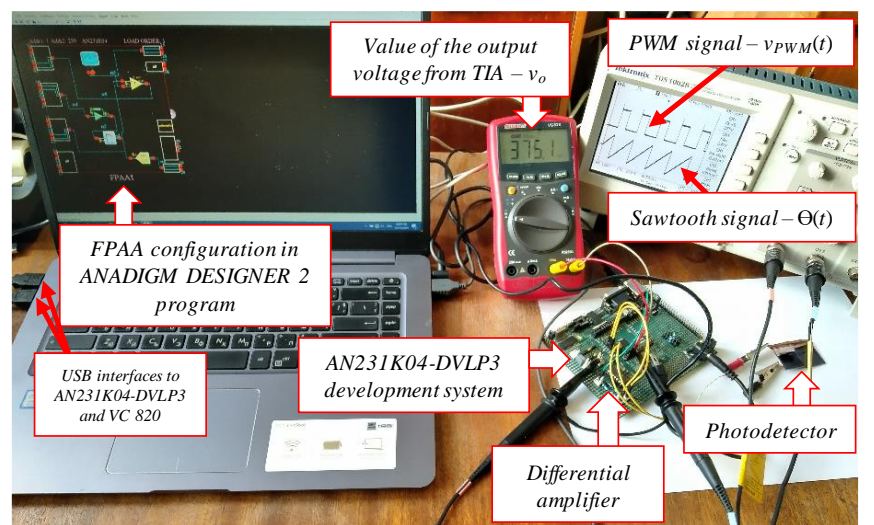

Fig. 10. Experimental setup for the prototype of the mixed-signal processing circuit integrated with the novel photodetector structure.

The testing of the PWM circuit was performed on light and dark using the fabricated photodetector. For this purpose, the maximum intensity of indoor light (in a laboratory with southern exposure in the time range from 10 am to $11 \mathrm{am}$ in December, without indoor lamp lightning) was greater than $450 \mathrm{~cd} / \mathrm{m}^{2}$. The dark condition was established with a plastic black box. Figure 11(a) and Figure 11(b) show the oscillograms of the sawtooth waves and the output pulse-width modulated signals at light and dark states, respectively. The average values of the output voltages were approximately $450 \mathrm{mV}$ and $78 \mathrm{mV}$, respectively. As can be seen, the amplitude of the sawtooth signal was $0.5 \mathrm{~V}$, as only $\theta^{+}(t)$ is shown. In addition, a question mark appeared next to the value of the pulse width, as it "floated" due to the constant change of the illumination in the laboratory.

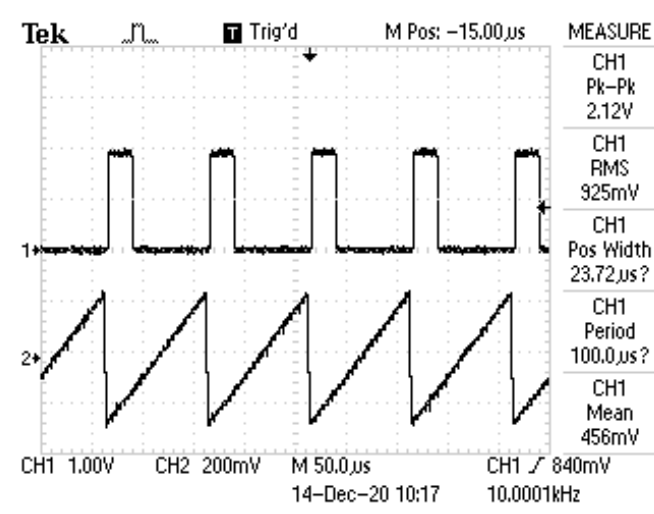

(a)

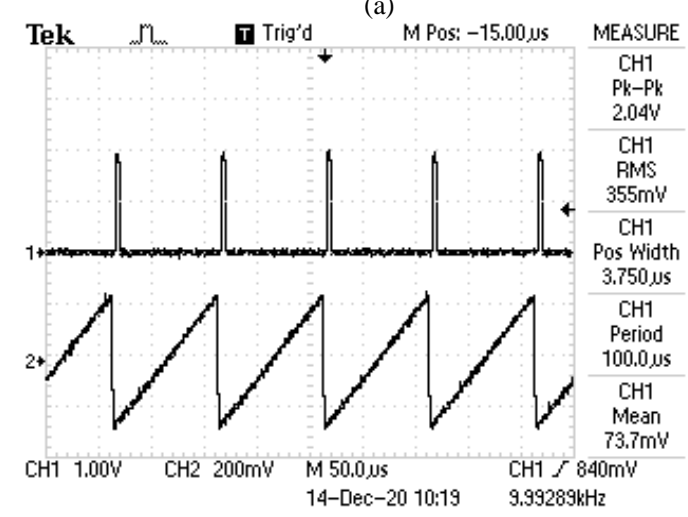

(b)

Fig. 11. Output waveforms $\left(v_{P W M}(t)-\mathrm{CH} 1\right.$ and $\left.\Theta(\mathrm{t})-\mathrm{CH} 2\right)$ of the PWM circuit: a) on light with intensity varied around $450 \mathrm{~cd} / \mathrm{m}^{2}$; b) on dark.

For the prototype, the transimpedance $z$ was selected equal to $200 \mathrm{k} \Omega$ (or $0.2 \mathrm{~V} / \mu \mathrm{A}$ ), and the amplitude of the sawtooth wave $\theta_{\max }$ was selected $1 \mathrm{~V}$. The comparative analysis between the defined parameters of the transfer coefficient $(\mathrm{z} \times \delta)$ and the experimentally obtained results showed that the relative error was below $1 \%$ with the change of the input current from $0.1 \mu \mathrm{A}$ up to $\approx 5 \mu \mathrm{A}$. At higher values of the input current, e.g., up to $10 \mu \mathrm{A}$, the $z$ had to be reduced to $100 \mathrm{k} \Omega$ or the $\theta_{\max }$ had to be increased to $2 \mathrm{~V}$.

\section{CONCLUSIONS}

In summary, a lead-free perovskite material was introduced to CdTe-based photoelectric thin film device for decreasing the absorbing layer thickness by keeping at the same time the performance parameters at levels competitive to the state-of-the-art detectors that use advanced photoelectric materials. The spectral sensitivity study of the new photodetector showed that it has good sensitivity in the blue region. The proposed microstructure was produced by conventional microfabrication technologies and was successfully integrated with a dynamic FPAA processing circuit, which exhibited a responsivity of $10.6 \mathrm{~mA} / \mathrm{W}$, response times of $30 \mathrm{~ms}$, and a non-linearity lower than $0.6 \%$. The module showed excellent sensitivity to slight light variations, resulting in pulse-width modulated signals at "light" to "dark" switching, including the smooth change of illuminance between the two states. This behavior makes the device suitable for application in precise position 
detection of smoothly moving objects. Future work will be related to an investigation of the long-term stability at outdoor conditions and to decrease the sensitive area by keeping the values of the performance parameters in the same range.

\section{CONFLICTS OF INTEREST}

The authors declare that they have no conflicts of interest.

\section{REFERENCES}

[1] J. Liu, Ch. Gao, L. Luo, Q. Ye, X. He, L. Ouyang, X. Guo, D. Zhuang, Ch. Liao, J. Meia, and W. Lau, "Low-temperature, solution processed metal sulfide as an electron transport layer for efficient planar perovskite solar cells", J. Mater. Chem. A, vol. 3, no. 22, pp 11750-11755, 2015. DOI: 10.1039/C5TA01200G.

[2] A. Acevedo-Luna, R. Bernal-Correa, J. Morites-Monsalve, and A. Morales-Acevedo, "Design of thin film solar cells based on a unified simple analytical model", Journal of Applied Research and Technology, vol. 15, no. 6, pp. 599-608, 2017. DOI: 10.1016/j.jart.2017.08.002.

[3] J. Li, J. Duan, X. Yang, Y. Duan, P. Yang, and Q. Tang, "Review on recent progress of lead-free halide perovskites in optoelectronic applications", Nano Energy, vol. 80, article 105526, 2021. DOI: 10.1016/j.nanoen.2020.105526.

[4] U. Khan, Y. Zhinong, A. A. Khan, A. Zulfiqar, and Q. Ullah khan, "Organic-inorganic hybrid perovskites based on methylamine lead halide solar cell”, Solar Energy, vol. 189, pp. 421-425, 2019. DOI: 10.1016/j.solener.2019.06.061.

[5] T. Nakamura, T. Handa, R. Murdey, Y. Kanemitsu, and A. Wakamiya, "Materials chemistry approach for efficient lead-free tin halide perovskite solar cells", ACS Appl. Electron. Mater., vol. 2, no. 12, pp. 3794-3804, 2020. DOI: 10.1021/acsaelm.0c00859.

[6] T. Ibn-Mohammed, S. C. L. Koh, I. M. Reaney, A. Acquaye, G. Schileo, K. B. Mustapha, and R. Greenough, "Perovskite solar cells: An integrated hybrid lifecycle assessment and review in comparison with other photovoltaic technologies", Renewable and Sustainable Energy Reviews, vol. 80, pp. 1321-1344, 2017. DOI: 10.1016/j.rser.2017.05.095.

[7] “Anadigm Product Range", Anadigm Corporate, Mesa, AZ, 85203, USA. [Online]. Available: https://www.anadigm.com

[8] R. Pawase and N. P. Futane, "MEMS seismic sensor with FPAAbased interface circuit for frequency-drift compensation using ANN", International Journal of Reconfigurable and Embedded Systems (IJRES), vol. 6, pp. 120-126, 2017. DOI: 10.11591/ijres.v6.i2.pp120126.

[9] R. Sanga, P. Sahoo, N. Murali, and S. A. V. Satya Murty, "Low power $\mathrm{pH}$ monitoring system using FPAA technology", in Proc. of International Conference in photonics, VLSI \& signal processing ICPVS - 2014, 2014, pp. 42-46.
[10] S. Shah and J. Hasler, "Low power speech detector on a FPAA", in Proc. of 2017 IEEE International Symposium on Circuits and Systems (ISCAS), Baltimore, MD, USA, 2017, pp. 1-4. DOI: 10.1109/ISCAS.2017.8050755.

[11] M. Balato, L. Costanzo, D. Gallo, C. Landi, M. Luiso, and M. Vitelli, "Design and implementation of a dynamic FPAA based photovoltaic emulator", Solar Energy, vol. 123, pp. 102-115, 2016. DOI: 10.1016/j.solener.2015.11.006.

[12] Y. Tian, K. Cai, D. Zhang, X. Liu, F. Wang, and B. Shirinzadeh, "Development of a XYZ scanner for home-made atomic force microscope based on FPAA control", Mechanical Systems and Signal Processing, vol. 131, pp. 222-242, 2019. DOI 10.1016/j.ymssp.2019.05.057.

[13] C. Ciofi, F. Crupi, C. Pace, G. Scandurra, and M. Patane, "A new circuit topology for the realization of very low-noise wide-bandwidth transimpedance amplifier", IEEE Transactions on Instrumentation and Measurement, vol. 56, no. 5, pp. 1626-1631, 2007. DOI: 10.1109/tim.2007.904566.

[14] E. Säckinger, "The transimpedance limit", IEEE Transactions on Circuits and Systems I: Regular Papers, vol. 57, no. 8, pp. 18481856, 2010. DOI: 10.1109/tcsi.2009.2037847.

[15] "AN-1803 design considerations for a transimpedance amplifier", Texas Instruments, Texas, Application Report SNOA515A-February 2008-Revised May 2013, May 2013. [Online]. Available: http://www.ti.com

[16] M. J. van der Horst, A. C. Linnenbank, W. A. Serdijn, and J. R. Long, "Systematic design of a transimpedance amplifier with specified electromagnetic out-of-band interference behavior", IEEE Transactions on Circuits and Systems I: Regular Papers, vol. 57, no. 3, pp. 530-538, 2010. DOI: 10.1109/tcsi.2009.2025003.

[17] S. M. Cox, C. K. Lam, and M. T. Tan, "A second-order PWMin/PWM-out class-D audio amplifier", IMA Journal of Applied Mathematics, vol. 78, no. 2, pp. 159-180, 2013. DOI: 10.1093/imamat/hxr042.

[18] C. K. Lam, M. T. Tan, S. M. Cox, and K. S. Yeo, "Class-D amplifier power stage with PWM feedback loop", IEEE Transactions on Power Electronics, vol. 28, no. 8, pp. 3870-3881, 2013. DOI: 10.1109/tpel.2012.2230027.

[19] D. Dobrilov and A. Tsolov, "Speed controller for DC motor," in Proc. of the Technical University of Sofia, 2016, vol. 66, book 1, pp. 315 320.

[20] AN231E04 Datasheet Rev. 1.5, $3^{\text {rd }}$ Generation. Dynamically Reconfigurable dpASP, Anadigm USA, 2007, 2021. [Online] Available: http://www.anadigm.com/_doc/DS231000-U001.pdf

[21] H. Fang and W. Hu, "Photogating in low dimensional photodetectors", Adv. Sci. (Weinh), vol. 4, no. 12, article 1700323 , 2017. DOI: $10.1002 /$ advs.201700323.

[22] Q. Liu, B. Cook, M. Gong, Y. Gong, D. Ewing, M. Casper, A. Stramel, and J. Wu, "Printable transfer-free and wafer-size MoS2/Graphene van der Waals heterostructures for high-performance photodetection", ACS Appl. Mater. Interfaces, vol. 9, no. 14, pp. 12728-12733, 2017. DOI: 10.1021/acsami.7b00912. 